Louisiana State University

LSU Digital Commons

Faculty Publications

Department of Entomology

$11-1-2011$

\title{
Horizontal transmission of Rickettsia felis between cat fleas, Ctenocephalides felis
}

\author{
Supanee Hirunkanokpun \\ School of Veterinary Medicine \\ Chutima Thepparit \\ School of Veterinary Medicine \\ Lane D. Foil \\ Louisiana State University \\ Kevin R. MacAluso \\ School of Veterinary Medicine
}

Follow this and additional works at: https://digitalcommons.Isu.edu/entomology_pubs

\section{Recommended Citation}

Hirunkanokpun, S., Thepparit, C., Foil, L., \& MacAluso, K. (2011). Horizontal transmission of Rickettsia felis between cat fleas, Ctenocephalides felis. Molecular Ecology, 20 (21), 4577-4586. https://doi.org/10.1111/ j.1365-294X.2011.05289.x

This Article is brought to you for free and open access by the Department of Entomology at LSU Digital Commons. It has been accepted for inclusion in Faculty Publications by an authorized administrator of LSU Digital Commons. For more information, please contact ir@lsu.edu. 


\title{
Horizontal transmission of Rickettsia felis between cat fleas, Ctenocephalides felis
}

\author{
Supanee Hirunkanokpun ${ }^{*}$, Chutima Thepparit ${ }^{*}$, Lane D. Foil ${ }^{\dagger}$, and Kevin R. Macaluso* \\ "Department of Pathobiological Sciences, School of Veterinary Medicine, Louisiana State \\ University, Skip Bertman Drive, SVM-3213, Baton Rouge, LA 70803, USA \\ tDepartment of Entomology, Louisiana State University, LSB-413, Baton Rouge, LA 70803, USA
}

\section{Abstract}

Rickettsia felis is a rickettsial pathogen primarily associated with the cat flea, Ctenocephalides felis. Although laboratory studies have confirmed that $R$. felis is maintained by transstadial and transovarial transmission in $C$. felis, distinct mechanisms of horizontal transmission of $R$. felis among cat fleas are undefined. Based on the inefficient vertical transmission of $R$. felis by cat fleas and the detection of $R$. felis in a variety of haematophagous arthropods, we hypothesize that $R$. felis is horizontally transmitted between cat fleas. Towards testing this hypothesis, flea transmission of $R$. felis via a bloodmeal was assessed weekly for 4 weeks. Rhodamine B was used to distinguish uninfected recipient and $R$. felis-infected donor fleas in a rickettsial horizontal transmission bioassay, and quantitative real-time PCR assay was used to measure transmission frequency; immunofluorescence assay also confirmed transmission. Female fleas acquired $R$. felis infection more readily than male fleas after feeding on a $R$. felis-infected bloodmeal for $24 \mathrm{~h}$ (69.3\% and $43.3 \%$, respectively) and both Rickettsia-uninfected recipient male and female fleas became infected with $R$. felis after cofeeding with $R$. felis-infected donor fleas (3.3-40.0\%). Distinct bioassays were developed to further determine that $R$. felis was transmitted from $R$. felisinfected to uninfected fleas during cofeeding and copulation. Vertical transmission of $R$. felis by infected fleas was not demonstrated in this study. The demonstration of horizontal transmission of $R$. felis between cat fleas has broad implications for the ecology of $R$. felis rickettsiosis.

\section{Keywords}

cat fleas; cofeeding; horizontal transmission; Rickettsia felis

\section{Introduction}

Rickettsia felis is an obligate intracellular Gram-negative bacterium belonging to the transitional group of Rickettsia (Gillespie et al.2007) and is the causative agent of an emerging flea-borne rickettsiosis (Azad et al. 1997). Although $R$. felis has been identified by molecular means in a large number of blood-feeding arthropods, only cat fleas,

Ctenocephalides felis, are associated with biological transmission of this pathogen (Azad et

\footnotetext{
(C) 2011 Blackwell Publishing Ltd

Correspondence: Kevin R. Macaluso, Fax: (225) 578 9701; kmacaluso@ vetmed.lsu.edu.

S.H. is a postdoctoral fellow, broadly interested in ecology of vector-borne pathogens and host-vector-pathogen interactions. K.R.M. is interested in the dynamics of rickettsial transmission by arthropods, molecular interaction between pathogen and vector, and mechanisms of vector competence.
}

Data accessibility: Raw data for gene copy numbers of Rickettsia felis 17-kDa and Ctenocephalides felis 18S rDNA in individual flea samples from rickettsial horizontal transmission bioassays are deposited at Dryad: doi:10.5061/dryad.vn88q. 
al. 1992; Reif \& Macaluso 2009). Cat fleas have a broad host range and, based on their ability to adapt to dynamic microenvironments, have a worldwide distribution.

Consequently, the occurrence of $R$. felis-infected cat fleas has been reported on a near cosmopolitan scale, and confirmed cases of flea-borne rickettsiosis caused by $R$. felis have been reported in seventeen countries to date (Reif \& Macaluso 2009; Ben-Zvi et al. 2010; Lindblom et al. 2010; Richards et al. 2010; Socolovsch et al. 2010; Williams et al. 2011).

Understanding the transmission of $R$. felis is essential to fully appreciate the epidemiology of this flea-borne rickettsiosis. The nature of interactions between different rickettsial species and their arthropod hosts is due in great part to the mode of transmission. This intricate relationship between Rickettsia and the arthropod host is best illustrated in the tickassociated spotted fever group of Rickettsia in which some rickettsial species not associated with vertebrate infection employ a maintenance strategy dependent on continued vertical, or inherited, transmission events (Niebylski et al. 1997). Conversely, virulent Rickettsia spp. with low efficiency of vertical transmission require frequent horizontal, or infectious, transmission events and often impose a negative fitness effect on the invertebrate host (Niebylski et al. 1999). Therefore, the pathogenicity of a particular rickettsial species is correlated to the necessity for successful horizontal transmission (Werren 1997). The complexity of the Rickettsia/arthropod relationship is highlighted by the dualistic nature of $R$. felis in cat fleas. Vertical transmission of $R$. felis has been described in laboratory-reared cat fleas (Azad et al. 1992; Wedincamp \& Foil 2002). Although the efficiency has yet to be precisely determined, it is clear that there is no $100 \%$ transovarial transmission, as evidenced by varying incidence of infection in several research colonies of cat fleas (Higgins et al. 1994; Reif \& Macaluso 2009). The variable vertical transmission of $R$. felis, coupled with compounding molecular and serological evidence of $R$. felis infection in a variety of arthropod and vertebrate hosts (Reif \& Macaluso 2009), suggests that $R$. felis possesses the characteristics of a rickettsial pathogen that also favours horizontal transmission. In nature, it is not known if $R$. felis is maintained primarily through vertical or horizontal transmission events; in fact, clear evidence of horizontal transmission has not been described.

Specific mechanisms of horizontal transmission including cofeeding, mating and contact with contaminated insect faeces that are known to occur for Rickettsia typhi, the flea-borne rickettsial agent of endemic typhus, have not been previously demonstrated in the $R$. felis/ arthropod model. Thus, the goal of the present study was to determine whether similar routes of transmission are involved in the spread of $R$. felis between fleas. Towards the objective, Rhodamine B (RB), which has previously been used as a biomarker for mammals, birds, fish and insects (Fisher 1999), was utilized to label cat fleas. Subsequently, bioassays were developed to assess horizontal transmission of $R$. felis between mixed populations of $R$. felis-infected donor and Rickettsia-uninfected recipient cat fleas. In addition to cofeeding infection of cat fleas, sexual transmission of $R$. felis was also demonstrated. The novel characterization of horizontal transmission of $R$. felis by cat fleas will further our understanding of this emerging rickettsiosis.

\section{Materials and methods}

\section{Source of fleas and Rickettsia}

Unfed, adult cat fleas ( $C$. felis) were purchased from Elward II (EL) (Soquel, CA, USA). Several generations of adult fleas were provided a Rickettsia-uninfected bovine (Rockland) bloodmeal via an artificial dog (Wade \& Georgi 1988), and eggs were reared to adults on sand with artificial diet (Lawrence \& Foil 2002). Cat fleas from the EL Laboratory are reportedly free of $R$. felis infection (Pornwiroon et al. 2007; Reif et al. 2011); however, a portion of these experimental cat fleas (30 male and 30 female) were tested for $R$. felis infection by quantitative real-time PCR (qPCR) to ensure the fleas were $R$. felis-free. An 
Ixodes scapularis-derived cell line (ISE6) maintained in modified L15B growth medium (Pornwiroon et al. 2006) was utilized to propagate $R$. felis (strain LSU). This rickettsial strain was originally isolated from the Louisiana State University cat flea colony (Pornwiroon et al. 2006). Cellular infection was examined by Diff-Quik (Dade Behring) staining as described previously by Pornwiroon et al. (2006); the BacLight viability stain kit (Invitrogen) was used to assess viability and enumerate rickettsiae as previously described (Sunyakumthorn et al. 2008).

\section{Rhodamine B labelling of fleas}

To differentiate $R$. felis-infected donor and uninfected recipient fleas in the rickettsial horizontal transmission bioassay, RB was evaluated as a biomarker in cat fleas. Newly emerged, unfed adult fleas were sexed, then male and female fleas, in separate feeding capsules, were allowed to prefeed with Rickettsia-uninfected heat-inactivated (HI) bovine blood for $24 \mathrm{~h}$. Five hundred microlitres of a $0.1 \%$ solution of RB (Sigma-Aldrich) in HI bovine serum was added to $100 \mu \mathrm{L}$ of bovine blood and offered to prefed fleas for $24 \mathrm{~h}$. Fleas were then fed on normal bovine blood for 30 days, and five of each RB-treated and control fleas were collected daily for RB detection by using a fluorescence stereomicroscope (Zeiss SteREO Lumar V12) at excitation wavelength $540 \mathrm{~nm}$ and emission wavelength 625 $\mathrm{nm}$.

\section{Detection of Rickettsia in fleas}

All flea samples were cleaned by sequential surface washing with $10 \%$ bleach for $5 \mathrm{~min}$, $70 \%$ ethanol for $5 \mathrm{~min}$ and three times ( $5 \mathrm{~min}$ each) sterile distilled water. Genomic DNA (gDNA) of each flea was extracted by a modified HotSHOT method (Truett et al. 2000; Reif et al. 2008). At least two negative environmental controls were performed during each DNA extraction process.

The standard plasmid, primers and reaction conditions for the qPCR used in this study have been described previously (Reif et al. 2008). Briefly, total $35 \mu \mathrm{L}$ of qPCR reaction contained $2 \times$ of iTaq SYBR Green Supermix with ROX (BioRad), 100 пм of each primer, DNase/RNase-free $\mathrm{dH}_{2} \mathrm{O}$, and $5 \mu \mathrm{L}$ of gDNA from each sample was premixed in 96-well plates and aliquoted in triplicate $10-\mu \mathrm{L}$ reactions on 384-well plates. Amplification and detection were performed with an ABI 7900HT unit (Applied Biosystems). For each set of reactions, two negative environmental controls for gDNA extraction and a negative control for the qPCR (ultrapure sterile water instead of template) were included. Additionally, for a portion of Rickettsia-positive fleas, gDNA was utilized for amplification and DNA sequencing of a 434-bp section the Rickettsia genus-specific 17-kDa antigen gene using the primers and protocol described by Pornwiroon et al. (2006). The sequences obtained were analysed with BioEdit sequence alignment editor (Ibis Biosciences), and nucleotide similarity comparisons were made with GenBank.

An indirect immunofluorescence assay (IFA) to detect $R$. felis infection was performed on both $R$. felis-infected donor and recipient fleas, using a modified protocol for booklice as described by Thepparit et al. (2011). Slides containing formalin-fixed paraffin-embedded flea sections $(4.5 \mu \mathrm{m})$ were heated at $65^{\circ} \mathrm{C}$ for $15 \mathrm{~min}$ and deparaffinized by repeated immersions in Hemo-De (Scientific Safety Solvents). Slides were rinsed with phosphatebuffered saline (PBS), and then antigen retrieval was performed by adding $10 \mu \mathrm{g} / \mathrm{mL}$ proteinase $\mathrm{K}$ (Roche Diagnostics) in prewarmed buffer containing 1 м Tris and 0.5 м EDTA $(\mathrm{pH} 8)$ at $37^{\circ} \mathrm{C}$ for $15 \mathrm{~min}$. At room temperature, the treated slides were excessively washed with PBS prior to immunofluorescence staining. For $R$. felis detection, mouse polyclonal antisera to $R$. felis were generated as described by Sunyakumthorn et al. (2008). The pretreated slides were blocked with $3 \%$ bovine serum albumin (BSA) in PBS for $1 \mathrm{~h}$. Then 
slides were incubated $2 \mathrm{~h}$ with mouse polyclonal anti- $R$. felis at a dilution of $1: 100$ in $1 \%$ BSA in PBS followed by three 5 min washes with PBS containing $0.01 \%$ TritonX-100. The slides were then incubated with fluorescein isothiocyanate (FITC)-conjugated goat antimouse IgG (KPL Inc.) at a dilution of 1:200 for $1 \mathrm{~h}$. After the slides were washed again with PBS/0.01\% Triton X-100, flea tissues were counterstained with $0.1 \%$ Evan's blue in PBS at $37{ }^{\circ} \mathrm{C}$ for 30 min. Finally, slides were mounted using Hardset Vectashield with DAPI (Vector Laboratories) and visualized using a Zeiss fluorescence microscope. Flea sections in which no primary antibody was added served as a control for nonspecific binding of the FITC-conjugated antibody.

\section{Rickettsial horizontal transmission bioassays}

For all transmission bioassays, newly emerged, unfed adult fleas were sexed and male and female fleas, in separate feeding capsules, were prefed with Rickettsia-uninfected HI bovine blood for $24 \mathrm{~h}$. Then, surviving fleas were starved of blood for 5-6 h prior to exposure to one of three different treatments: (i) $R$. felis-infected donor, (ii) RB-labelled uninfected recipient or (iii) uninfected recipient fleas. For the first treatment, an $R$. felis-infected bloodmeal was prepared by resuspending intact $R$. felis-infected cells containing $5 \times 10^{9}$ rickettsiae (passage 7) and collecting the cells by centrifugation at $13000 \mathrm{~g}$ for $10 \mathrm{~min}$. Culture medium was removed, and the $R$. felis-infected cell pellet was resuspended in 600 $\mu \mathrm{L}$ of $\mathrm{HI}$ bovine blood. Both prefed male and female fleas in separate capsules were allowed to feed on the $R$. felis-infected bloodmeal for $24 \mathrm{~h}$. For the second treatment, fleas were RBlabelled as described earlier. The third treatment consisted of HI bovine blood in the absence of rickettsiae or $\mathrm{RB}$.

Four groups (I-IV) were assessed for rickettsial transmission between fleas utilizing fleas from $R$. felis-infected donor, RB-labelled uninfected recipient, and uninfected control and recipient flea feeding treatments (Fig. 1A). Group I consisted of $80 R$. felis-infected donor male fleas placed in the same feeding capsule with 80 uninfected recipient female fleas. Group II was reciprocal and consisted of 80 uninfected recipient male fleas placed in the same feeding capsule with $80 R$. felis-infected donor female fleas. In Group III, 80 each of $R$. felis-infected donor male and female fleas were placed in the same feeding capsule with 80 each of RB-labelled uninfected recipient male and female fleas. The control group (Group IV) was comprised of 80 each of uninfected male and female fleas in the same feeding capsule. Fleas in all four groups were fed on normal bovine blood, replenished every other day for 28 days, using the artificial feeding system. Ten male and female fleas were assessed for $R$. felis infection at days $1,7,14,21$ and 28 post-cofeeding. For Groups I, II and IV, fleas were collected and sexed, and the remaining fleas were placed back into their feeding capsules. For Group III, fleas were sexed and then RB-labelled uninfected recipient fleas were distinguished from $R$. felis-infected donor fleas by fluorescent microscopy. All fleas were kept at $-20^{\circ} \mathrm{C}$ until gDNA was extracted. The remaining flea eggs with faeces in each feeding capsule on days $3,7,14,21$ and 28 were reared until $F_{1}$ adults emerged (approximately 20-25 days). Five each of male and female $F_{1}$ fleas from each experimental group were assessed for rickettsial infection by qPCR. Three separate trials were conducted, and data presented are a combination of the three trials.

To pinpoint the mechanisms of horizontal transmission, the contributions of cofeeding and mating were examined in two separate trials for each route. The data are presented as a combination of the two trials. Cofeeding bioassays (Fig. 1B) were executed by combining 80 each of $R$. felis-infected donor male and female fleas into a feeding capsule and allowing them to feed on $2 \mathrm{~mL}$ of uninfected bovine blood for $24 \mathrm{~h}$. A $200 \mu \mathrm{L}$ portion of the leftover blood was collected and assessed for $R$. felis infection by qPCR; meanwhile, the remaining blood was immediately offered to uninfected recipient female (Group V) and male (Group VI) fleas for $24 \mathrm{~h}$. New, uninfected bovine blood $(2 \mathrm{~mL})$ was again provided twice more to 
R. felis-infected donor fleas for $24 \mathrm{~h}$ and then, again, $200 \mu \mathrm{L}$ of leftover blood was processed by qPCR, and the remaining blood was offered to Group V and VI fleas. At all times, the uninfected recipient female (Group V) and male (Group VI) fleas were separated to prevent mating. Ten each of Group V and VI fleas were collected at 24, 48 and $72 \mathrm{~h}$ postfeeding on the initial leftover bovine blood and assessed for $R$. felis infection.

For the mating bioassays (Fig. 1C), newly emerged, unfed adult fleas were sexed and male and female fleas held in separate feeding capsules. Newly emerged male and female fleas do not mate prior to a bloodmeal, and feeding for approximately $24 \mathrm{~h}$ is required for successful mating, with most fleas mating by $48 \mathrm{~h}$ (Bi-song et al. 2002). Therefore, 80 each of virgin male and female fleas were prefed with Rickettsia-uninfected HI bovine blood for $24 \mathrm{~h}$, starved for 5-6 h and then $R$. felis-exposed for $24 \mathrm{~h}$. In Group VII, $80 R$. felis-infected donor males were placed in the same feeding capsule with 80 uninfected recipient females. For Group VIII, 80 uninfected recipient males were placed in the same feeding capsule with 80 $R$. felis-infected donor females. To eliminate cofeeding transmission between $R$. felisinfected and uninfected fleas, both groups were allowed to mate in the absence of a bloodmeal. Ten male and female fleas from each group were assessed for $R$. felis infection at 12,36 and $60 \mathrm{~h}$ post-mating.

\section{Statistical analysis}

Statistical analyses were performed using the GLM procedure of SAS ${ }^{\circledR}$ version 9.1.3 (SAS Institute Inc). Two-way analysis of variance (ANova) was performed to examine potential differences of $R$. felis acquisition, transmission and density (ratio of $R f 17 \mathrm{kDa} / C f 18 \mathrm{~S}$ copy numbers). If overall significance was observed, Tukey's honestly significant difference post hoc test was used to determine pairwise differences of means of main effects. For significant interaction effects, pairwise comparisons were conducted with a $t$-test of least squares means. Differences among or between fleas and bioassay groups were considered statistically significant at $P<0.05$.

\section{Results}

\section{Rhodamine B-labelled fleas}

All adult fleas fed RB-labelled serum were luminescent when examined by fluorescent microscopy. In comparison with unlabelled control fleas fed bovine blood alone, fleas exposed to RB-labelled bloodmeal appeared orange on day 1 post-feeding, with most of the body emitting signal (Fig. 2). At 7 days, and for up to 30 days, post-feeding on a single RBlabelled blood-meal, signal from the RB label was only detected in the eyes of fleas (Fig. 2, in the white circles). Survival of fleas fed RB-labelled bloodmeal was not different from control fleas. Using RB-labelled bloodmeal allowed for differentiation of fleas from unlabelled control fleas; thus, the RB-labelled fleas could be utilized to examine horizontal transmission of $R$. felis between fleas in the subsequent bioassay.

\section{Infection of fleas with $\boldsymbol{R}$. felis and transmission to previously uninfected fleas}

A portion of EL colony of fleas (30 males and 30 females) were confirmed $R$. felis negative by qPCR prior to utilization in rickettsial transmission bioassays. Fleas were exposed to $R$. felis-infected bloodmeal, RB-labelled bloodmeal or bloodmeal alone for $24 \mathrm{~h}$, and then divided into one of four groups. Fleas in each Group (I-IV) were allowed to cofeed for 28 days; $R$. felis infection was assessed by qPCR in individual fleas at days 1, 7, 14, 21 and 28 post-cofeeding (Table 1). Both donor male and female fleas were $R$. felis positive beginning 1 day after feeding on the $R$. felis-infected bloodmeal. The mean prevalence for all fleas $( \pm \mathrm{SEM})$ of $R$. felis acquisition by female fleas $(69.3 \pm 2.7 \%)$ exposed to the $R$. felis feeding treatment was significantly greater than male fleas $(43.3 \pm 4.7 \%)$. 
Both uninfected recipient male and female fleas became positive for $R$. felis as evidenced by qPCR after cofeeding with $R$. felis-infected donor fleas (Table 1). Differences between sexes were not identified based on group assignment. In Group I, mean $R$. felis acquisition by recipient females over 28 days was 4.7\% (7/150) after being combined with $R$. felisinfected donor males. A total of $16 \%$ (24/150) of uninfected recipient male fleas combined with $R$. felis-infected donor female fleas in Group II became positive for $R$. felis for the same period of time. For Group III, 10.3\% (31/300) of both RB-labelled uninfected recipient male and female fleas became positive for $R$. felis. Over the course of cohabitation by $R$. felis-infected donor and recipient fleas, no significant difference in the mean percent transmission of $R$. felis to either recipient male $(13.7 \pm 2.7 \%)$ or female $(7.0 \pm 1.5 \%)$ fleas was identified; however, the mean prevalence of $R$. felis in recipient fleas was significantly lower than $R$. felis-infected donor male and female fleas. The transmission of $R$. felis from $R$. felis-infected donor fleas to uninfected recipient fleas in each Group (I-III) was confirmed by traditional PCR using the $17-\mathrm{kDa}$ antigen gene primer sets (Williams et al. 1992; Pornwiroon et al. 2006) and sequencing amplicons from a representative subsample of $R$. felis-positive recipient fleas. Nucleotide sequences for the amplified fragments (394 bp) of $17-\mathrm{kDa}$ antigen gene were identical to the published sequences for $R$. felis (LSU) (Pornwiroon et al. 2006) and $R$. felis URRWXCal2 (Accession no. CP000053) (Ogata et al. 2005). All unlabelled, unexposed control male and female fleas (Group IV), fed normal bovine blood, were qPCR negative for $R$. felis.

Further confirmation of $R$. felis transmission by $R$. felis-infected donor fleas to uninfected recipient fleas was demonstrated by IFA. While IFA confirmed that uninfected recipient fleas became infected after being reared with $R$. felis-infected donor fleas (Fig. 3A-C), it was obvious that the recipient fleas had lower $R$. felis density (Fig. 3D,E), when compared with $R$. felis-infected donor fleas. Control (Group IV) fleas fed on normal bovine blood were IFA negative (inset rectangle in Fig. 3A).

Genomic equivalents of the $R$. felis $17-\mathrm{kDa}$ antigen gene and the $C$. felis $18 \mathrm{~S}$ rDNA gene were quantified, and the density of $R$. felis in individual fleas was determined by calculating the ratio of the logarithmically transformed $R$. felis $17-\mathrm{kDa}$ to $18 \mathrm{~S}$ rDNA copy number. When comparing the density of $R$. felis between $R$. felis-infected donor male and female fleas, the mean $R f 17 \mathrm{kDa} / C f 18 \mathrm{~S}$ ratio of $R$. felis-infected donor females (0.75) was significantly greater than the same ratio in $R$. felis-infected donor male (0.58) fleas (Fig. 4). When combining results from Groups I-III, no significant difference in mean $R f 17 \mathrm{kDa} /$ $C f 18$ S ratios between recipient male (0.32) and female (0.37) fleas (Fig. 4) was observed. There were significant differences in rickettsial density between fleas in the $R$. felis-infected donor cohort compared to those that became infected with $R$. felis after they were placed in the same feeding capsule (Fig. 4). Likewise, the mean $R f 17 \mathrm{kDa} / C f 18 \mathrm{~S}$ ratio of $R$. felisinfected donor fleas was significantly increased from day $1-7$ post-cofeeding, remaining significantly greater at all other collection time points compared to day 1 post-cofeeding (Fig. 5). There were no significant differences in mean $R f 17 \mathrm{kDa} / C f 18 \mathrm{~S}$ ratios among recipient fleas collected at different time points (day 1-28 post-cofeeding) (Fig. 5). Total rickettsial infection load was also determined in both $R$. felis-infected donor and recipient fleas by quantifying the copy number of $R f 17 \mathrm{kDa}$ per individual flea lysate. Rickettsia felisinfected donor fleas had very low $R$. felis infection load at day $1\left(7.8 \times 10^{3}\right)$, increasing significantly on day 7 through day $28\left(7.4 \times 10^{6}-1.3 \times 10^{7}\right.$, respectively). There was no significant difference in $R$. felis infection load among recipient fleas from day 1-28 (6.9 $\times$ $10^{2}-5.6 \times 10^{3}$, respectively) after cofeeding with the $R$. felis-infected donor fleas.

\section{Vertical transmission of $\boldsymbol{R}$. felis to $\mathrm{F}_{\mathbf{1}}$ fleas was not detected}

After $R$. felis-infected donor and uninfected recipient fleas were placed together in different horizontal transmission Groups (I-III) as previously described, female fleas from each 
Group laid eggs. Their eggs, not separated from faecal material, from days 3, 7, 14, 21 and

28 were transferred into separate petri dishes containing sand and flea larval diet, and allowed to develop to the $F_{1}$ adult stage. A subsample of $F_{1}$ adults from each group were tested for $R$. felis infection by qPCR. Of 450 fleas examined, none of $\mathrm{F}_{1}$ adult fleas were positive for $R$. felis.

\section{Cofeeding and mating contribute to horizontal transmission of $\boldsymbol{R}$. felis by fleas}

During the previous rickettsial horizontal transmission bioassay, combining $R$. felis-infected donor and uninfected recipient fleas in the same feeding capsule allowed for both cofeeding and mating to occur. To further define the specific mechanisms of horizontal transmission, the contributions of cofeeding and mating were examined separately (Fig. 1). For cofeeding transmission potential, fleas were exposed to $R$. felis and subsequently exposed to new $R$. felis-free bloodmeal, from which a portion of each new bloodmeal was assessed for $R$. felis infection and offered to Rickettsia-uninfected fleas. Rickettsia felis DNA was detected in leftover blood at 24 and, again at $48 \mathrm{~h}$ post-exposure $\left(3.3 \times 10^{3}\right.$ and $3.0 \times 10^{2}$ rickettsiae per $200 \mu \mathrm{L}$ of leftover blood, respectively). Subsequently, 5.8\% (7/120) of Rickettsia-uninfected fleas that fed on this leftover blood also became $R$. felis positive over $72 \mathrm{~h}$ of exposure. The number of $R$. felis-positive recipient females (five fleas) was greater than recipient males (two fleas). However, the mean $R f 17 \mathrm{kDa} / C f 18 \mathrm{~S}$ ratio in recipient males $(0.38)$ was greater than females (0.31).

To determine the ability of fleas to transmit $R$. felis during mating, fleas were exposed to $R$. felis and subsequently combined with uninfected recipient fleas in a capsule in the absence of a bloodmeal, again separated out by sex, and 10 fleas each from Group VII (female) and Group VIII (male) were assessed for $R$. felis infection at 12,36 and $60 \mathrm{~h}$ postmating. Over two trials, approximately $3.3 \%$ (4/120) of recipient fleas became $R$. felis positive after being combined with $R$. felis-infected donor fleas. Similar to cofeeding, the number of $R$. felispositive recipient females (three fleas) was greater than males (one flea). Likewise, the mean $R f 17 \mathrm{kDa} / C f 18 \mathrm{~S}$ ratio in the recipient male $(0.39)$ was greater than females $(0.30)$.

\section{Discussion}

Epidemics of insect-borne rickettsial pathogens such as louse-borne Rickettsia prowazekii are typically associated with extreme living conditions including overcrowding, famine and war (Azad 1988). Throughout history, these pathogens have accounted for severe morbidity and mortality (Azad \& Beard 1998). In the last 20 years, only one new insect-borne rickettsial pathogen, $R$. felis, has been described. A closer examination of the ecology and epidemiology of $R$. felis is needed to assess the public health importance of this emerging pathogen. Although $R$. felis is associated with a variety of arthropods including several blood-feeding arthropods, for example ticks, mites and multiple species of fleas (Reif \& Macaluso 2009), cat fleas are the only known hematophagous vector of $R$. felis (Azad et al. 1992; Reif \& Macaluso 2009). Interestingly, recent studies have identified and characterized a biological relationship between $R$. felis in non-hematophagous psocids (booklice) in which $R$. felis is a true endosymbiont (Perotti et al. 2006; Behar et al. 2010; Thepparit et al. 2011). The movement of Rickettsia species between widely distinct arthropods, conferring differential host-dependent fitness effects, is known to occur (Himler et al. 2011; Jiggins \& Hurst 2011); thus, the infection of booklice is not unexpected. However, the symbiotic role of $R$. felis in psocids contrasts the horizontal transmission potential established with hematophagous arthropods associated with recent foci of human infection (Socolovsch et al. 2010; Williams et al. 2011) with clinical symptoms similar to other rickettsial diseases including fever, headache and general morbidity (Reif \& Macaluso 2009). Epidemiological studies of this emerging insect-borne rickettsial pathogen have been hindered by the gap in 
our understanding of the precise mechanisms of transmission to vertebrates and among invertebrate hosts.

Horizontal transmission of arthropod-borne Rickettsia occurs by multiple mechanisms including cofeeding, mating and contact with contaminated faeces (Hayes et al. 1980; Azad 1990; Matsumoto et al. 2005). In this study, we show that $R$. felis can be transmitted between cat fleas by both cofeeding and mating. Laboratory studies have indicated that $R$. felis is maintained in cat fleas by vertical transmission (transovarial and transstadial transmission). The efficiency of transmission is dependent on several still unknown factors, accounting for varying prevalence in colonized populations ranging from $0 \%$ to $100 \%$ (Reif $\&$ Macaluso 2009). Horizontal and vertical transmission of $R$. felis by wild-caught fleas has not been proven. The potential for horizontal transmission of $R$. felis to vertebrate hosts is supported by the identification of $R$. felis in the salivary glands of cat fleas (Macaluso et al. 2008 ), detection of $R$. felis DNA in the blood of cats exposed to $R$. felis-infected fleas, and subsequent host response to $R$. felis exposure by seroconversion (Wedincamp \& Foil 2000). Despite this circumstantial evidence of arthropod vector-to-vertebrate host transmission cycle, transmission of $R$. felis between arthropods had not been demonstrated. This study is the first account of distinct horizontal transmission of $R$. felis between infected and uninfected fleas. The infectious dose used in current study was previously employed to generate an $R$. felis-infected cohort at incidence ranging 58-69\% (Reif et al. 2011). Cat fleas exposed to $R$. felis via the artificial host system served as donor fleas and able to transmit the agent to uninfected recipient fleas when mixed together. Our results in the cofeeding bioassay demonstrated that $R$. felis was released from infected fleas to the bloodmeal during feeding. Moreover, uninfected fleas also became infected with $R$. felis after feeding on this leftover blood. While transmission of $R$. felis by bite of infected fleas is likely, as $R$. felis has been identified in salivary glands of the cat fleas (Macaluso et al. 2008), the kinetics and specific mechanisms of transmission via flea feeding require further investigation.

The previously undefined kinetics of horizontal transmission were examined in the current study demonstrating $R$. felis DNA in both donor and recipient fleas 1 day post-cofeeding in the same feeding capsule, suggesting a rapid expansion of infection to various tissues of the fleas. The transmission of $R$. felis to vertebrate hosts, in the absence of flea faeces, resulting in the induction of an antibody response has been demonstrated (Wedincamp \& Foil 2000). Thus, while introduction of $R$. felis to vertebrate hosts via flea feeding has been shown, the acquisition of $R$. felis by naive cofed fleas was not known. The transmission of $R$. felis between arthropods via cofeeding on vertebrate blood has broad implications towards infection and potential transmission by cat fleas and other hematophagous arthropods. Upon exposure, if dissemination and infection of $R$. felis to flea ovaries or salivary glands occur, then cofeeding transmission of $R$. felis could greatly expand the number of potential vectors within foci. Conversely, if $R$. felis infection does not result in subsequent competent vectors, then surveillance of potential vectors of this pathogen should not be based solely upon detection of the rickettsiae in sampled arthropod populations. Over 19 arthropod species have been shown to be positive for R felis DNA via PCR assay (Reif \& Macaluso 2009); their role as vectors remains undefined.

Sexual transmission of Rickettsia species has been described for other hematophagous arthropods. For example, transmission of pathogenic Rickettsia rickettsii from infected male to uninfected female Ixodes ricinus ticks has been described (Hayes et al. 1980). In the current study in the absence for the potential for cofeeding infection, $R$. felis transmission from donor to recipient fleas at 12 and $36 \mathrm{~h}$ postmating was demonstrated. This may account for significant transmission of $R$. felis as cat fleas are known to have multiple mating events (Hsu \& Wu 2000). Cat fleas mated on a cat host have significantly longer mating duration time when compared with off-host mating (Hsu \& Wu 2001); thus, the observed low 
transmission efficiency may be due to other factors including the density of rickettsiae and duration of mating as fleas were mated off-host (in the absence of bloodmeal).

Consequently, the low transmission efficiency observed in the current study may explain why previous examination of transmission of $R$. felis by copulation or direct contact including cofeeding between infected and uninfected cat fleas failed to detect horizontal transmission events (Wedincamp \& Foil 2002). As discussed previously, studies on the infection of ovaries or salivary glands in female fleas occurring subsequent to venereal transmission will be required to properly assess the importance of this route of horizontal transmission by cat fleas in the epidemiology of $R$. felis.

Even though vertical transmission is thought to be the primary mechanism of maintenance of $R$. felis by colonized cat fleas (Azad et al. 1992; Reif \& Macaluso 2009), we did not detect vertical transmission of $R$. felis from fleas infected as adults to their respective progeny. As the primary objective of this study was to assess horizontal transmission, only a small proportion of $\mathrm{F}_{1}$ fleas from the different experimental groups were examined for $R$. felis infection. For example, testing over 450 individuals, $F_{1}$ represented only $0.43 \%$ of the total $F_{1}$ produced; thus, missing a vertical transmission event is possible. A focused study to examine transovarial transmission efficiency by horizontally infected fleas should be conducted to complete the portrait of the $R$. felis transmission cycle.

In summary, this study demonstrates the first evidence of horizontal transmission of $R$. felis between cat fleas; both cofeeding and mating are mechanisms involved in the horizontal transmission of $R$. felis. In combination with known vertical transmission, maintenance and spread of $R$. felis between flea hosts are enhanced by horizontal transmission. The specifics of transmission are essential to understanding the ecology of $R$. felis in nature, and additional studies are needed to further evaluate other potential vectors of $R$. felis, as well as the mechanisms of transmission of $R$. felis to humans.

\section{Acknowledgments}

We thank M.L. Guillotte, P. Sunyakumthorn, K.E. Reif, T.M. Mascari and J.A. Macaluso for comments and technical assistance. We also thank M. Kearney for help with statistical analysis. This work was supported by NIH $\mathrm{AI} 069248$ (S1) and AI077784.

\section{References}

Azad, AF. Relationship to vector biology and epidemiology of louse and flea-borne rickettsioses. In: Walker, DH., editor. Biology of Rickettsial Diseases. CRC Press; Boca Raton, Florida: 1988. p. 52-62.

Azad AF. Epidemiology of murine typhus. Annual Review of Entomology. 1990; 35:553-569.

Azad AF, Beard CB. Rickettsial pathogens and their arthropod vectors. Emerging Infectious Diseases. 1998; 4:179-186. [PubMed: 9621188]

Azad AF, Sacci JB Jr, Nelson WM, et al. Genetic characterization and transovarial transmission of a typhus-like rickettsia found in cat fleas. Proceedings of the National Academy of Sciences, USA. 1992; 89:43-46.

Azad AF, Radulovic S, Higgins JA, Noden BH, Troyer JM. Flea-borne rickettsioses: ecologic considerations. Emerging Infectious Diseases. 1997; 3:319-327. [PubMed: 9284376]

Behar A, McCormick LJ, Perlman SJ. Rickettsia felis infection in a common household insect pest, Liposcelis bostrychophila (Psocoptera: Liposcelidae). Applied and Environmental Microbiology. 2010; 76:2280-2285. [PubMed: 20139311]

Ben-Zvi I, Meltzer E, Nogueras MM, Segura F, Bank I. First detection of human infection with Rickettsia felis in Israel. American Journal of the Medical Sciences. 2010; 340:343. [PubMed: 20881761] 
Bi-song W, Fang-dong Z, Qi-zhi S, Jing L. Mating behavior of the cat flea, Ctenocephalides felis Bouche (Siphonaptera: Pulicidae) and male response to female extract on an artificial feeding system. Entomologia Sinica. 2002; 9:29-43.

Fisher P. Review of using Rhodamine B as a marker for wildlife studies. Wildlife Society Bulletin. 1999; 27:318-329.

Gillespie JJ, Beier MS, Rahman MS, et al. Plasmids and rickettsial evolution: insight from Rickettsia felis. PLoS One. 2007; 2:e266. [PubMed: 17342200]

Hayes SF, Burgdorfer W, Aeschlimann A. Sexual transmission of spotted fever group rickettsiae by infected male ticks: detection of rickettsiae in immature spermatozoa of Ixodes ricinus. Infection and Immunity. 1980; 27:638-642. [PubMed: 7380544]

Higgins JA, Sacci JB Jr, Schriefer ME, Endris RG, Azad AF. Molecular identification of rickettsia-like microorganisms associated with colonized cat fleas (Ctenocephalides felis). Insect Molecular Biology. 1994; 3:27-33. [PubMed: 8069413]

Himler AG, Adachi-Hagimori T, Bergen JE, et al. Rapid spread of a bacterial symbiont in an invasive whitefly is driven by fitness benefits and female bias. Science. 2011; 332:254-256. [PubMed: 21474763]

Hsu MH, Wu WJ. Effects of multiple mating on female reproductive output in the cat flea (Siphonaptera: Pulicidae). Journal of Medical Entomology. 2000; 37:828-834. [PubMed: 11126536]

Hsu MH, Wu WJ. Off-host observations of mating and postmating behaviors in the cat flea (Siphonaptera: Pulicidae). Journal of Medical Entomology. 2001; 38:352-360. [PubMed: 11372958]

Jiggins FM, Hurst GD. Microbiology. Rapid insect evolution by symbiont transfer. Science. 2011; 332:185-186. [PubMed: 21474745]

Lawrence W, Foil LD. The effects of diet upon pupal development and cocoon formation by the cat flea (Siphonaptera: Pulicidae). Journal of Vector Ecology. 2002; 27:39-43. [PubMed: 12125871]

Lindblom A, Severinson K, Nilsson K. Rickettsia felis infection in Sweden: report of two cases with subacute meningitis and review of the literature. Scandinavian Journal of Infectious Diseases. 2010; 42:906-909. [PubMed: 20735330]

Macaluso KR, Pornwiroon W, Popov VL, Foil LD. Identification of Rickettsia felis in the salivary glands of cat fleas. Vector-Borne and Zoonotic Diseases. 2008; 8:391-396. [PubMed: 18399779]

Matsumoto K, Ogawa M, Brouqui P, Raoult D, Parola P. Transmission of Rickettsia massiliae in the tick, Rhipicephalus turanicus. Medical and Veterinary Entomology. 2005; 19:263-270. [PubMed: 16134974]

Niebylski ML, Schrumpf ME, Burgdorfer W, et al. Rickettsia peacockii sp. nov., a new species infecting wood ticks, Dermacentor andersoni, in western Montana. International Journal of Systematic Bacteriology. 1997; 47:446-452. [PubMed: 9103635]

Niebylski ML, Peacock MG, Schwan TG. Lethal effect of Rickettsia rickettsii on its tick vector (Dermacentor andersoni). Applied and Environmental Microbiology. 1999; 65:773-778. [PubMed: 9925615]

Ogata H, Renesto P, Audic S, et al. The genome sequence of Rickettsia felis identifies the first putative conjugative plasmid in an obligate intracellular parasite. PLoS Biology. 2005; 3:e248. [PubMed: 15984913]

Perotti MA, Clarke HK, Turner BD, Braig HR. Rickettsia as obligate and mycetomic bacteria. FASEB Journal. 2006; 20:2372-2374. [PubMed: 17012243]

Pornwiroon W, Pourciau SS, Foil LD, Macaluso KR. Rickettsia felis from cat fleas: isolation and culture in a tick-derived cell line. Applied and Environmental Microbiology. 2006; 72:5589-5595. [PubMed: 16885313]

Pornwiroon W, Kearney MT, Husseneder C, Foil LD, Macaluso KR. Comparative microbiota of Rickettsia felis-uninfected and -infected colonized cat fleas, Ctenocephalides felis. ISME Journal. 2007; 1:394-402. [PubMed: 18043659]

Reif KE, Macaluso KR. Ecology of Rickettsia felis: a review. Journal of Medical Entomology. 2009; 46:723-736. [PubMed: 19645274] 
Reif KE, Stout RW, Henry GC, Foil LD, Macaluso KR. Prevalence and infection load dynamics of Rickettsia felis in actively feeding cat fleas. PLoS One. 2008; 3:e2805. [PubMed: 18665265]

Reif KE, Kearney MT, Foil LD, Macaluso KR. Acquisition of Rickettsia felis by cat fleas during feeding. Vector-Borne and Zoonotic Diseases. 2011; 11:963-968. [PubMed: 21214386]

Richards AL, Jiang J, Omulo S, et al. Human Infection with Rickettsia felis, Kenya. Emerging Infectious Diseases. 2010; 16:1081-1086. [PubMed: 20587178]

Socolovsch C, Mediannikov O, Sokhna C, et al. Rickettsia felis-associated uneruptive fever, Senegal. Emerging Infectious Diseases. 2010; 16:1140-1142. [PubMed: 20587190]

Sunyakumthorn P, Bourchookarn A, Pornwiroon W, et al. Characterization and growth of polymorphic Rickettsia felis in a tick cell line. Applied and Environmental Microbiology. 2008; 74:3151-3158. [PubMed: 18359823]

Thepparit C, Sunyakumthorn P, Guillotte ML, et al. Isolation of a rickettsial pathogen from a nonhematophagous arthropod. PLoS One. 2011; 6:e16396. [PubMed: 21283549]

Truett GE, Heeger P, Mynatt RL, et al. Preparation of PCR-quality mouse genomic DNA with hot sodium hydroxide and Tris (HotSHOT). BioTechniques. 2000; 29:52-54. [PubMed: 10907076]

Wade SE, Georgi JR. Survival and reproduction of artificially fed cat fleas, Ctenocephalides felis Bouche (Siphonaptera: Pulicidae). Journal of Medical Entomology. 1988; 25:186-190. [PubMed: 3392715]

Wedincamp J Jr, Foil LD. Infection and seroconversion of cats exposed to cat fleas (Ctenocephalides felis Bouche) infected with Rickettsia felis. Journal of Vector Ecology. 2000; 25:123-126. [PubMed: 10925806]

Wedincamp J Jr, Foil LD. Vertical transmission of Rickettsia felis in the cat flea (Ctenocephalides felis Bouche). Journal of Vector Ecology. 2002; 27:96-101. [PubMed: 12125879]

Werren JH. Wolbachia run amok. Proceedings of the National Academy of Sciences, USA. 1997; 94:11154-11155.

Williams SG, Sacci JB Jr, Schriefer ME, et al. Typhus and typhus like rickettsiae associated with opossums and their fleas in Los Angeles County, California. Journal of Clinical Microbiology. 1992; 30:1758-1762. [PubMed: 1629332]

Williams M, Izzard L, Graves SR, Stenos J, Kelly JJ. First probable Australian cases of human infection with Rickettsia felis (cat flea typhus). Medical Journal of Australia. 2011; 194:41-43. [PubMed: 21449868] 

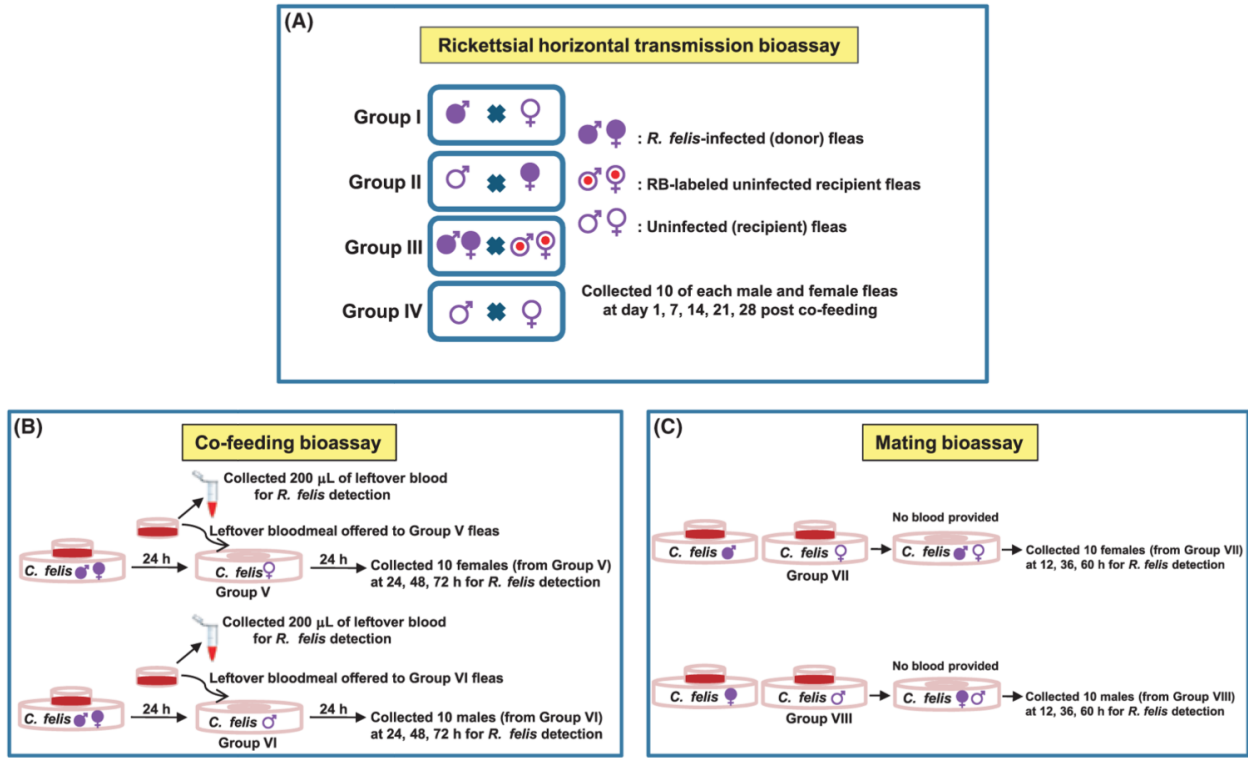

Fig. 1.

Diagrammatic representation of three rickettsial transmission bioassays. (A) The experimental design for rickettsial horizontal transmission bioassay consisted of four groups (I-IV); rickettsial transmission between fleas from Rickettsia felis-infected donor fleas, and Rhodamine B-labelled uninfected recipient or uninfected recipient fleas was assessed. (B) The experimental design for the co-feeding bioassay via the bloodmeal from $R$. felisinfected donor to uninfected recipient fleas. (C) Experimental design for the mating bioassay from $R$. felis-infected donor to uninfected recipient fleas. 


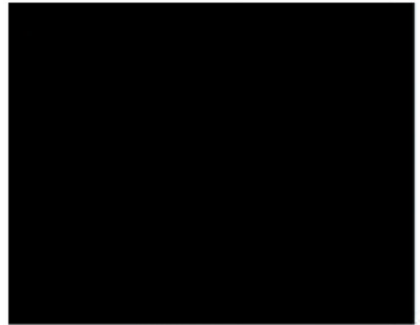

Control

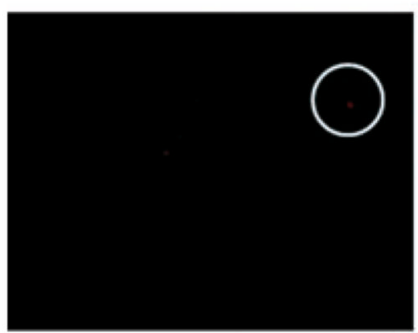

Day 14

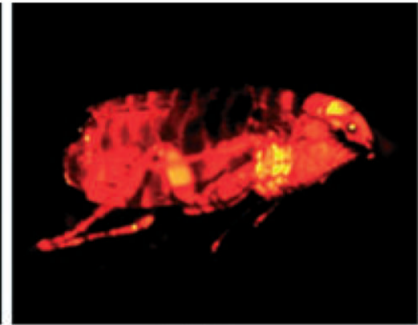

Day 1

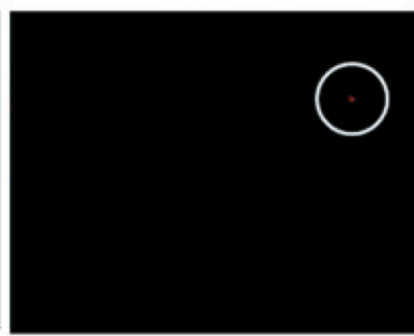

Day 21

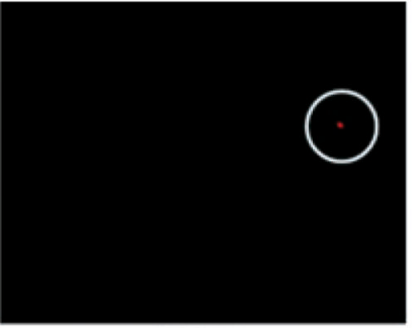

Day 7

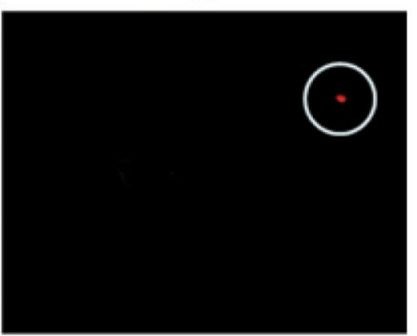

Day 30

Fig. 2.

Fluorescence microscopy images of adult fleas after feeding on Rhodamine B (RB). A representative control flea, pictured in the top left column, fed on normal bovine blood. Other columns show RB-labelled fleas collected at days 1, 7, 14, 21 and 30 post-feeding on $0.1 \%$ Rhodamine B-labelled bloodmeal. 


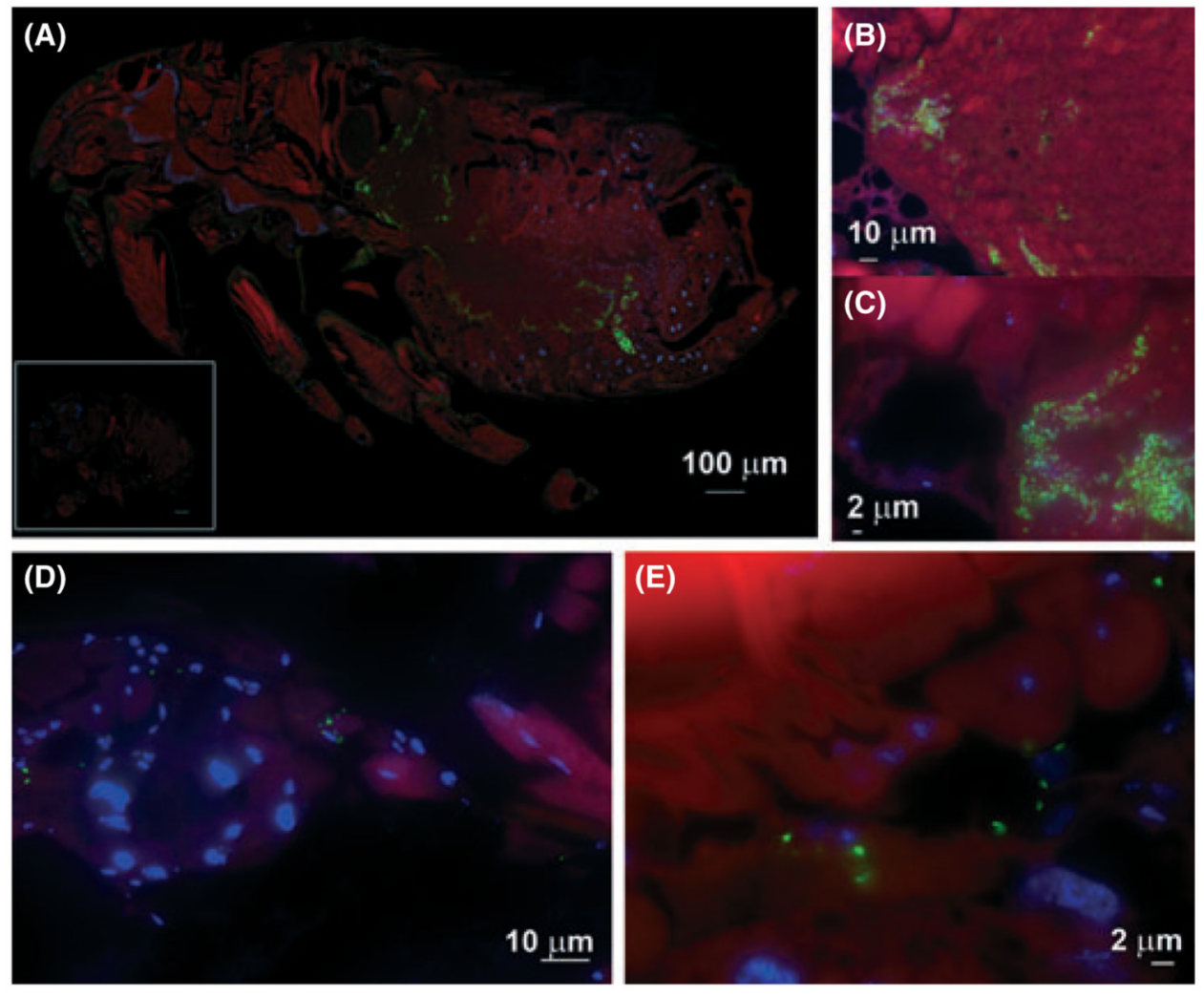

Fig. 3.

Immunofluorescence detection of Rickettsia felis in donor and recipient fleas. Fleas were exposed to $5 \times 10^{9}$ rickettsiae and infection visualized at day 28 post-cofeeding at (A) $10 \times$, (B) $40 \times$ and (C) 100×. Recipient fleas cofed with $R$. felis-infected donor fleas also became infected as seen at (C) $40 \times$ and (D) $100 \times$. The inset rectangle in (A) is a control uninfected flea fed on normal bovine blood. 


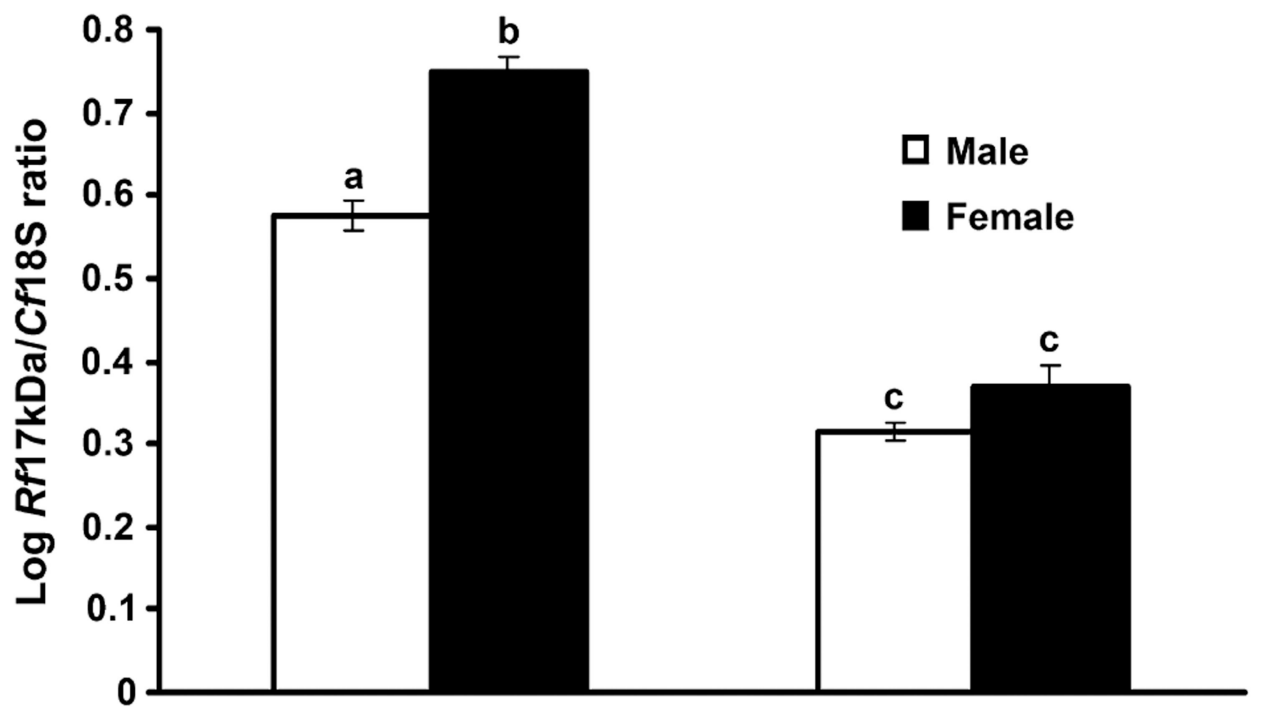

R. felis-infected donor fleas

Recipient fleas

Fig. 4.

Overall mean Rickettsia felis infection density of male and female $R$. felis-infected donor and recipient fleas. Rickettsial densities in male and female $R$. felis-infected donor and recipient fleas in Groups I-III (combined) were assessed by qPCR. Donor female fleas had the greatest density of $R$. felis, compared with donor male fleas and both sexes of recipient fleas. Bars represent means $( \pm \mathrm{SEM})$ of $R f 17 \mathrm{kDa} / C f 18 \mathrm{~S}$ ratio; means with different letters are significantly different. 


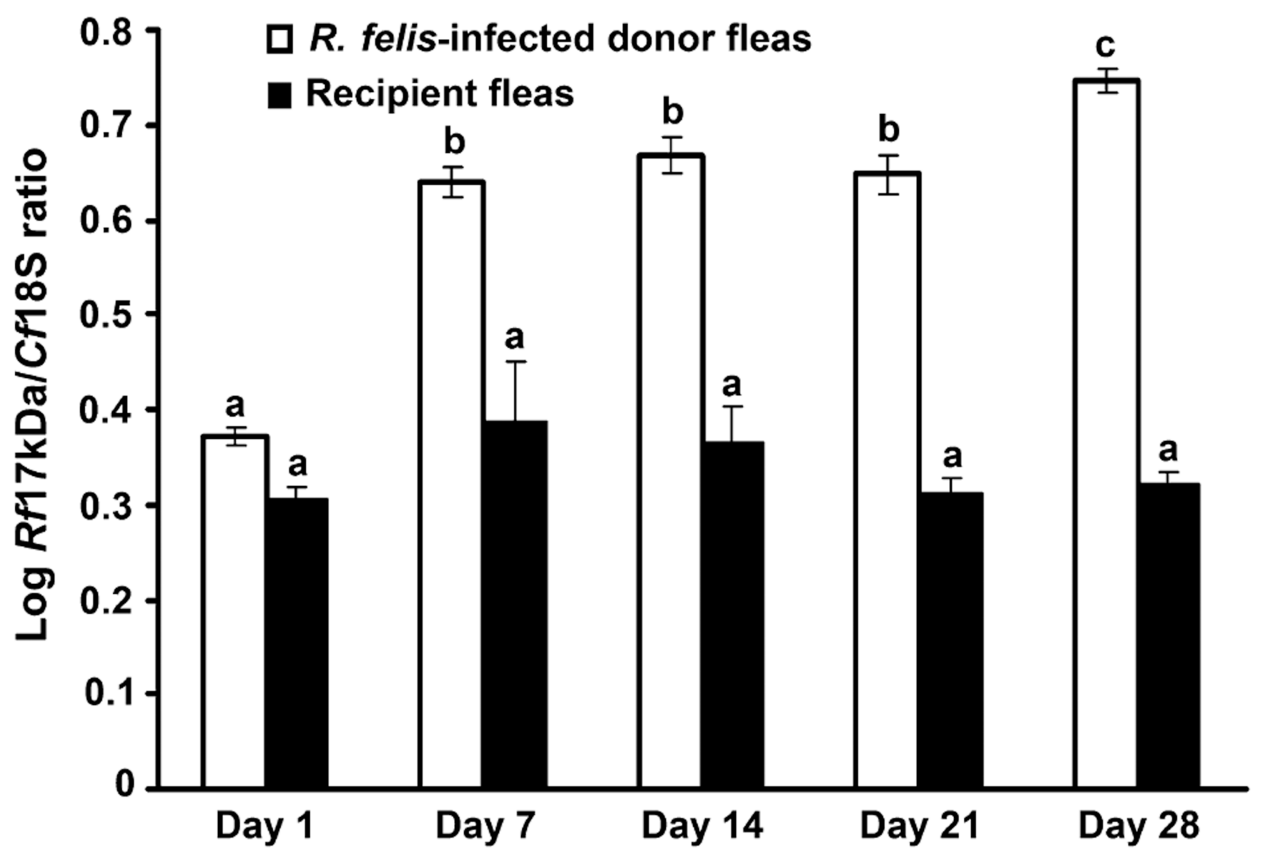

Fig. 5.

Mean Rickettsia felis infection density of $R$. felis-infected donor and recipient fleas at different time points. Rickettsial densities in $R$. felis-infected donor and recipient fleas collected at days $1,7,14,21$ and 28 post-cofeeding were assessed by qPCR. In a temporal fashion, donor fleas typically had greater densities, compared with recipient fleas. Bars represent means $( \pm \mathrm{SEM})$ of $R f 17 \mathrm{kDa} / \mathrm{C} f 18 \mathrm{~S}$ ratio; means with different letters are significantly different. 


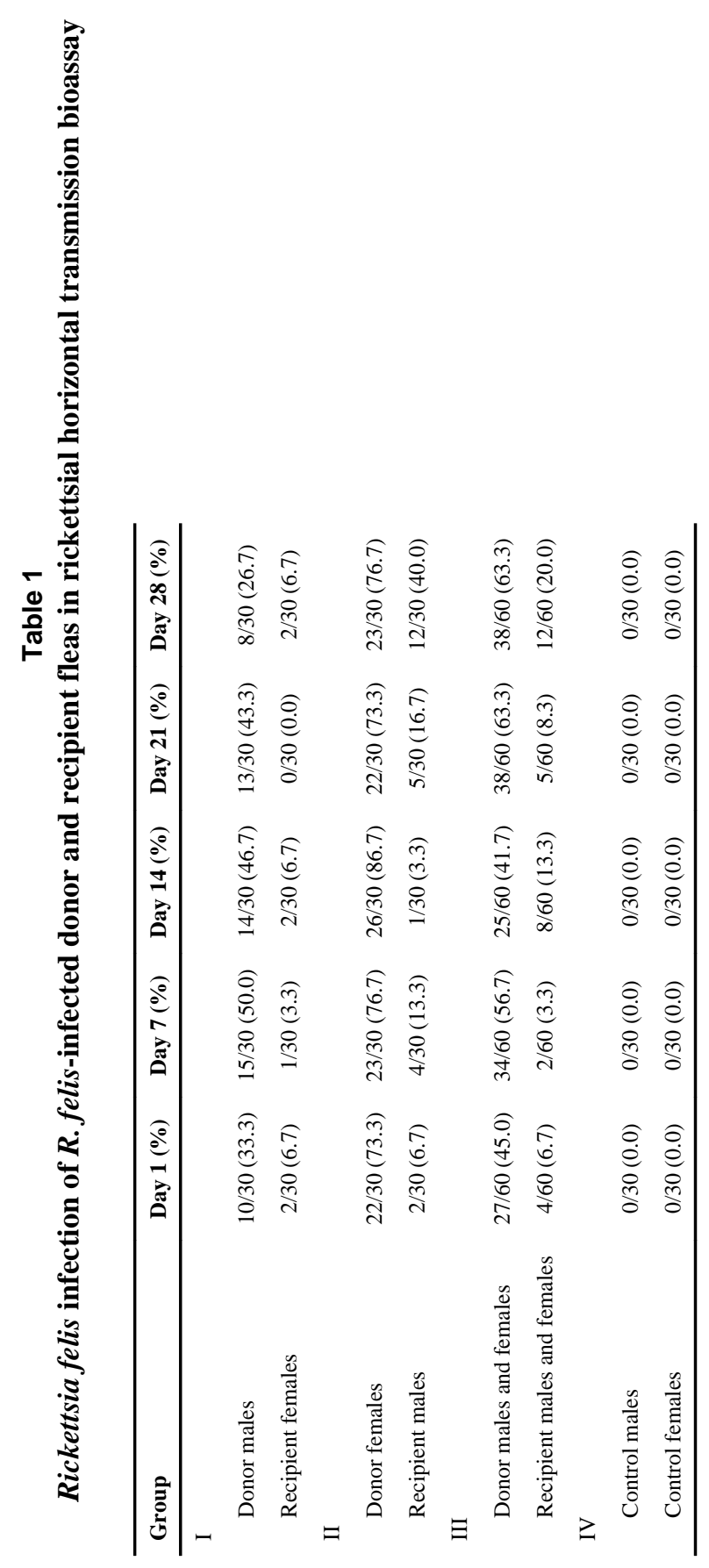

Mol Ecol. Author manuscript; available in PMC 2013 August 11. 